\title{
Experimental Study on Mesoscopic Shear Behavior of Calcareous Sand Material with Digital Imaging Approach
}

\author{
Jianhua Shen, ${ }^{1}$ Xing Wang $\mathbb{D}^{1},{ }^{1}$ Wenbai Liu, ${ }^{2,}$ Poyu Zhang, ${ }^{1}$ Changqi Zhu, ${ }^{1}$ \\ and Xinzhi Wang ${ }^{1}$ \\ ${ }^{1}$ State Key Laboratory of Geotechnical Mechanics and Engineering, Institute of Rock and Soil Mechanics, \\ Chinese Academy of Sciences, Wuhan 430071, China \\ ${ }^{2}$ College of Ocean Science and Engineering, Shanghai Maritime University, Shanghai 201306, China
}

Correspondence should be addressed to Xing Wang; wangxing1213@hotmail.com

Received 7 September 2020; Revised 26 October 2020; Accepted 4 November 2020; Published 23 November 2020

Academic Editor: Loke Foong

Copyright (C) 2020 Jianhua Shen et al. This is an open access article distributed under the Creative Commons Attribution License, which permits unrestricted use, distribution, and reproduction in any medium, provided the original work is properly cited.

\begin{abstract}
The study of the mesostructure of soil under loading is the basis for understanding its macromechanical properties and for establishing its constitutive model. In this study, a series of shear tests was performed on dry calcareous sand under constant normal stress by a modified direct shear apparatus. Digital images of the sample at different shear stages are obtained. The mesostructural parameters of the sample are then extracted and analyzed using an image analysis technique. The results show that the shear-band is located at the junction of the upper and lower shear boxes with a thickness of 0.79-1.59 mm. During shearing, the position of the maximum shear strain incremently shifted to the junctions between the two shear boxes. The azimuths of the particles prior to the test distribute symmetrically on both sides of $90^{\circ}$. After the test, the azimuths of the particles are mainly obtuse angles $\left(150-180^{\circ}\right)$ and the long axis of the particles generally points in the opposite direction from the shear-band. The sand particles undergo four stages: random arrangement during initial sample preparation, compaction under normal stress, particle rotation during shearing, and ordered alignment after shearing. The test results help to reveal the movement mechanism of calcareous sand at the mesoscopic level during the direct shear process.
\end{abstract}

\section{Introduction}

Calcareous sand is a kind of marine sediment with the calcium carbonate content generally exceeding $50 \%$. It has the characteristics of brittle fractures, high porosity, and high angularity, which make its mechanical behavior significantly different from those of the continental sediments [1]. There have been numerous studies on the engineering characteristics of calcareous sand at the macroscopic level. However, the related research on the mechanical behavior of calcareous sand at the mesoscopic level is quite limited [2-6]. Although the mesostructural parameters including particle geometry and internal porosity of calcareous sand have been studied by many researchers, the relationship between these parameters and the macromechanical behavior is unclear. It has been found that the evolution of the mesostructure is the fundamental factor determining the changes in the macroscopic physical and mechanical properties of soils [7]. The mesostructure is the basis for the construction of a structural physical model of the soil and also a key point for solving complex geotechnical problems $[8,9]$. Therefore, it is of great importance to perform an indepth study of the mesostructure changes of calcareous sand under loading.

Since Terzaghi proposed the paradigm of microstructure and mesostructure of soils, numerous studies have been conducted on the microstructure and mesostructure using the methods such as photoelastic material $[10,11]$, sectioning $[12,13], \mathrm{X}$-rays $[14,15]$, and CT scanning $[16,17]$. Due to the limitations of the test equipment, testing techniques, and analytical methods, it is difficult to measure the microscopic and mesoscopic performance of the specimen under loading. Therefore, the true characteristics and appearance of the geotechnical materials during tests have not 
been revealed [18]. With the development of computer technology [19-23], the application of digital image processing technology in the study of the mesostructure of soils has become increasingly mature. For example, Alshibli and Sture [24] analyzed the thicknesses of shear-band via digital image processing technology. White et al. [25] studied the deformation of the sand during the sinking process using digital image processing technology. Li et al. [26, 27] studied the deformation fields of foundations under loading using the digital images processing software. Likewise, Zhou et al. $[28,29]$ performed visualized cyclic triaxial tests to study the liquefaction of sand and measured mesoscopic parameters of sand using digital image processing technology. Zhang et al. [30], Shao et al. [31], and Liu and Tian [32] conducted various mechanical tests and studied the mesostructure of the sand particles using digital image processing techniques. In addition, Chen et al. [33], Zhang et al. [34], Divya et al. [35], Pang et al. [36], Safavizadeh et al. [37], Lyu et al. [38], Bowman and Haigh [39], Wang et al. [40], Mou et al. [41], Liu et al. [42], Yu et al. [43], Xu et al. [44], Li et al. [45], and Chen et al. [46] used digital image processing techniques to study the mechanical properties of geotechnical materials from different perspectives.

Although previous scholars have also carried out a lot of research work on the variation of the mesostructure of particles during the direct shear, most of these results are limited to terrigenous quartz sand. Calcareous sand has a special mineral composition, irregular particle shape, and high void ratio, which are significantly different from terrigenous quartz sand. Whether the previous research results are suitable for calcareous sand needs further study. The aim of this study is to measure mesostructural parameters of calcareous sand in the direct shear test by using digital image processing technology. The deformation characteristics of calcareous sand were then analyzed from a microscopic perspective. Firstly, the methods for the extraction and analysis of the mesostructural parameters were summarized. Then, direct shear tests were conducted on calcareous medium sand by a modified direct shear apparatus with digital photography having the visualization feature. Finally, the mesostructural parameters were extracted from the digital photos by means of image analysis software and analyzed at different shearing stages. The test results could aid the understanding of the shearing process of calcareous sand at the mesoscopic level.

\section{Methodology}

2.1. Experimental Materials. The calcareous sand used in this study was collected from a reclaimed island-reef in the South China Sea (Figure 1(a). The sample site is located in the shallow surface layer downstream of the fill site, and it is approximately $5.0 \mathrm{~m}$ from the ground (Figure 1(c). During the dredging construction process (Figure 1(b), the movement of the particles is affected by particle weight and water flow. The coarse particles are firstly deposited at the bottom of the stratum, which is close to the dredging inlet, while the fine particles flow with the water to the downstream of the dredging inlet and are silted at the top of the stratum. During the foundation treatment, the coarse particles which are accumulated at the dredging inlet are often pushed downstream. Due to the intermittent alternation of the dredging and the foundation treatment, the coarse and fine particles are finally distributed alternately in the middle of the stratum. As a result, the strength of soil among different layers varies.

In this study, the calcareous medium sand with the particle size of $0.5-1.0 \mathrm{~mm}$ was used in order to avoid the scale effect on the experiment results and to obtain more distinguishable digital image processing results. Before the test, the calcareous sand was firstly extracted from the coarse-grained soil layer, as shown in Figure 1(c). Then the sand was air-dried and the $0.5-1.0 \mathrm{~mm}$ particles were obtained by the standard sieving test. Because of the weak cementation of the calcareous sand, a small number of finegrained particles adhere to the surfaces of the tested particles. During the direct shear test, these fine particles might be removed from the surfaces of the coarse particles, which could, in turn, affect the results of digital image processing. In order to ensure the reliability of the digital image processing results, the $0.5-1.0 \mathrm{~mm}$ sized calcareous sand was rinsed with clean water prior to the direct shear test. Finally, the sand was oven-dried to conduct the direct shear tests. The physical parameters of the sample are shown in Table 1.

2.2. Experimental Equipment. A half-mold direct shear apparatus (Figure 2(a)) built from the ZQB-4 light-weight direct shear instrument which is produced by the Nanjing Soil Instrument Factory, China, was used in this study. Half of the upper and lower shear boxes were cross-cut and replaced by two plexiglass plates with a thickness of $2.5 \mathrm{~mm}$ to allow the real-time monitoring of the movement of the sand particles during shearing. In order to avoid any distortion of the test results induced by the excessive friction between the two plexiglass plates during shearing, a thin layer of lubricant was applied at the contact area of the two plexiglass plates before the test. The detailed structure of this instrument could be found in the patent by Liu et al. [47]. The modified ZQB-4 light-weight direct shear apparatus has external dimensions of $850 \mathrm{~mm} \times 550 \mathrm{~mm} \times 1100 \mathrm{~mm}(L \times$ $W \times H)$ and a total weight of $40 \mathrm{~kg}$. To avoid large lateral deformation of the transparent plexiglass plates under normal stress, the maximum normal stress allowed by the modified ZQB-4 direct shear apparatus was $400 \mathrm{kPa}$. The lever ratio was $1: 20$ and the normal stress was applied by the dead weight. The cross-sectional area of the sample of the modified ZQB-4 direct shear apparatus was reduced to half of the original area (i.e., $15 \mathrm{~cm}^{2}$ ), so the normal stress of $400 \mathrm{kPa}$ could be achieved by a weight of $5.1 \mathrm{~kg}$. The horizontal shear force could be as high as $1.2 \mathrm{kN}$, which is measured by the proving ring with a dial gauge. The proving ring was calibrated by a national metrology agency before the test, and the relationship between the force, $F(\mathrm{kN})$, and the dial gauge reading, $n(\mathrm{~mm})$, was obtained. During shearing, the position of the upper shear box is fixed, and the lower shear box is moved towards the proving ring. The displacement and shear rate of the sample are controlled by 


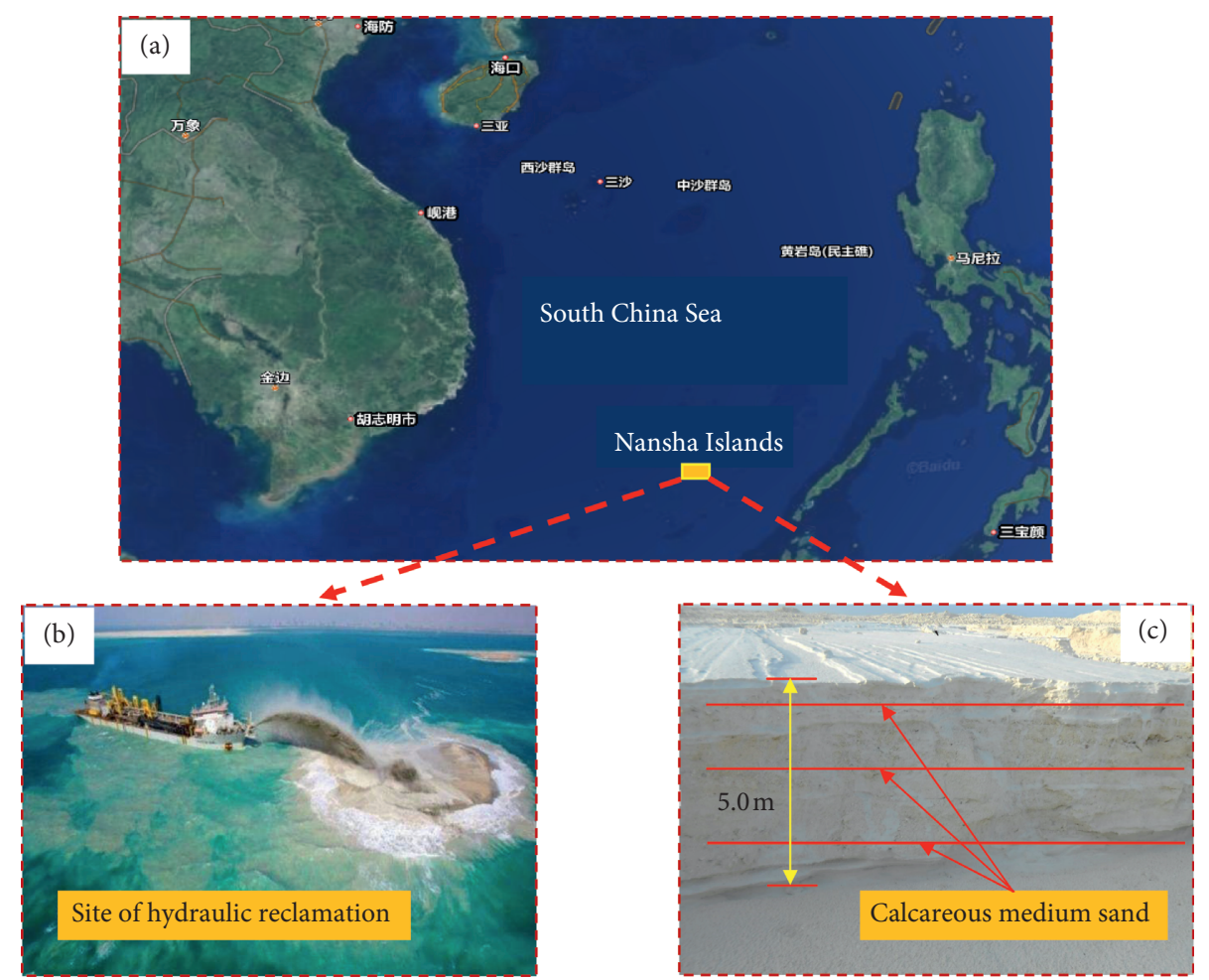

FIgURE 1: Location of the study area. (a) Location of Nansha Islands; (b) site of hydraulic reclamation; (c): sampling sites [1].

turning a handwheel. A shear displacement of $0.2 \mathrm{~mm}$ is achieved by each turn of the handwheel.

The mesostructural parameters of the sample were extracted by the image acquisition system which consists of a camera apparatus, a Bluetooth camera controller, and a tripod as shown in Figure 2(b). The camera used in this study had a resolution of 12 million pixels, which is high enough to meet the requirements of mesostructural analysis of particle images. The tripod is a VCT-5208RM tripod produced by China Zhongshan Yunteng Photographic Equipment Co., Ltd and a mobile phone clip that could be rotary horizontally and is vertically fixed at the head of the tripod to mount the camera. The mobile phone camera is mounted close to the transparent plexiglass plates by the tripod, and the positions of the sand particles are recorded within a fixed time interval or shear displacement (Figure 2(b)). After the test, the images are sequentially exported from the memory of the mobile phone and analyzed with an image analysis software to extract the mesostructural parameters, which are difficult to obtain in the conventional direct shear tests. The Bluetooth camera controller allows real-time image capturing of the sand particles without touching the camera, which ensures that the distance between the camera and the sample remains constant. The technical parameters of the components of the image acquisition system are shown in Table 2.

2.3. Testing Procedures. Half-mold direct shear tests were carried out on dry calcareous sand to investigate the changes of the mesostructural properties of sand particles during shearing. Via field survey of the reclaimed coral reef, the degree of compaction of the soil (i.e., the ratio of dry density to maximum dry density) after the foundation treatment is generally greater than $90 \%$. Besides, the low-rise buildings are the most common construction form on the island; thus, the basement stresses are mostly within $200-300 \mathrm{kPa}$. The basement stresses of the few high-rise structures (i.e., signal towers, maritime lighthouses, etc.) do not exceed $400 \mathrm{kPa}$ [48-50]. Therefore, the degree of compaction and normal stress were set as $90 \%$ and $400 \mathrm{kPa}$, respectively. The wet calcareous sand particles are darker in the image due to the humidity, which makes it difficult to distinguish the particles from the intergranular pores in the sample. In order to facilitate the processing of digital images in this study, the direct shear tests were performed under dry conditions.

In order to eliminate the impact of the height of the sample on the test results, the porous stones laid on the top and bottom sides of the sample used in the conventional direct shear test were removed so that the height of the sample was $4.0 \mathrm{~cm}$. The shearing mode was set to consolidated-quickly shearing and the shear rate of $0.8 \mathrm{~mm} / \mathrm{min}$ was used. The test was terminated when the shear displacement reached $10.0 \mathrm{~mm}$. The experiment design parameters are shown in Table 3.

The test process was divided into three stages: sample preparation, loading (or consolidation), and shearing, which are illustrated as follows:

(1) Before the test, calcareous sand was oven-dried at $105^{\circ} \mathrm{C}$, cooled in a drying dish to room temperature (about $25^{\circ} \mathrm{C}$ ). Then, the mass of the sample was calculated based on the degree of compaction (90\%) and the volume of the sample $\left(60 \mathrm{~cm}^{3}\right)$. The sample was then evenly placed into the shear box by the 
TABle 1: Physical parameters of the calcareous sand tested.

\begin{tabular}{|c|c|c|c|c|c|c|c|c|}
\hline Sample & $\begin{array}{c}\text { Particle size } \\
D(\mathrm{~mm})\end{array}$ & $\begin{array}{l}\text { Dry density } \\
\rho_{d}\left(\mathrm{~g} / \mathrm{cm}^{3}\right)\end{array}$ & $\begin{array}{c}\text { Water } \\
\text { content } \omega \\
(\%)\end{array}$ & $\begin{array}{l}\text { Specific } \\
\text { gravity Gs }\end{array}$ & $\begin{array}{l}\text { Minimum dry } \\
\text { density } \rho_{d \text { min }} \\
\left(\mathrm{g} / \mathrm{cm}^{3}\right)\end{array}$ & $\begin{array}{l}\text { Maximum dry } \\
\text { density } \rho_{d \max } \\
\left(\mathrm{g} / \mathrm{cm}^{3}\right)\end{array}$ & $\begin{array}{l}\text { Min void } \\
\text { ratio } e_{\text {min }}\end{array}$ & $\begin{array}{l}\text { Max void } \\
\text { ratio } e_{\max }\end{array}$ \\
\hline $\begin{array}{l}\text { Calcareous } \\
\text { sand }\end{array}$ & $0.5-1.0$ & 1.05 & 0 & 2.78 & 0.86 & 1.17 & 1.38 & 2.23 \\
\hline
\end{tabular}

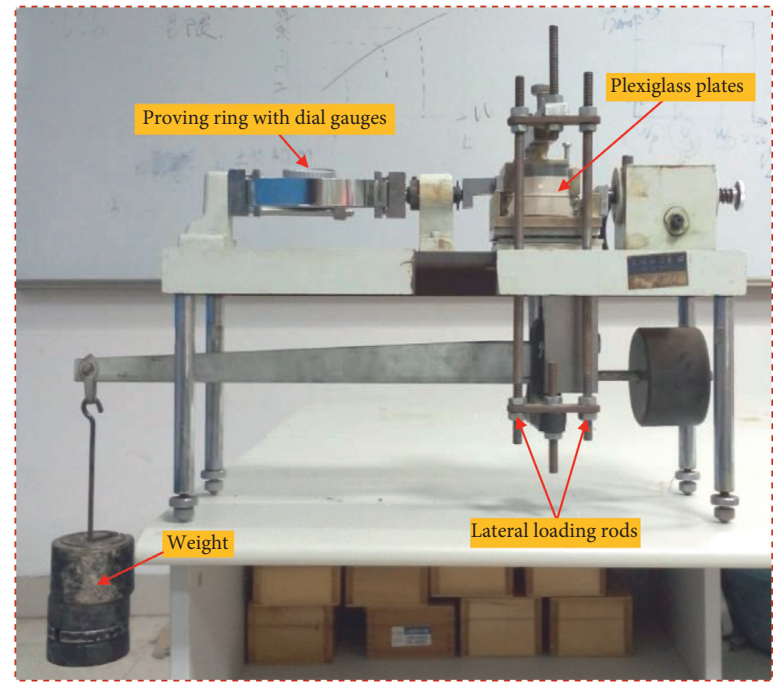

(a)

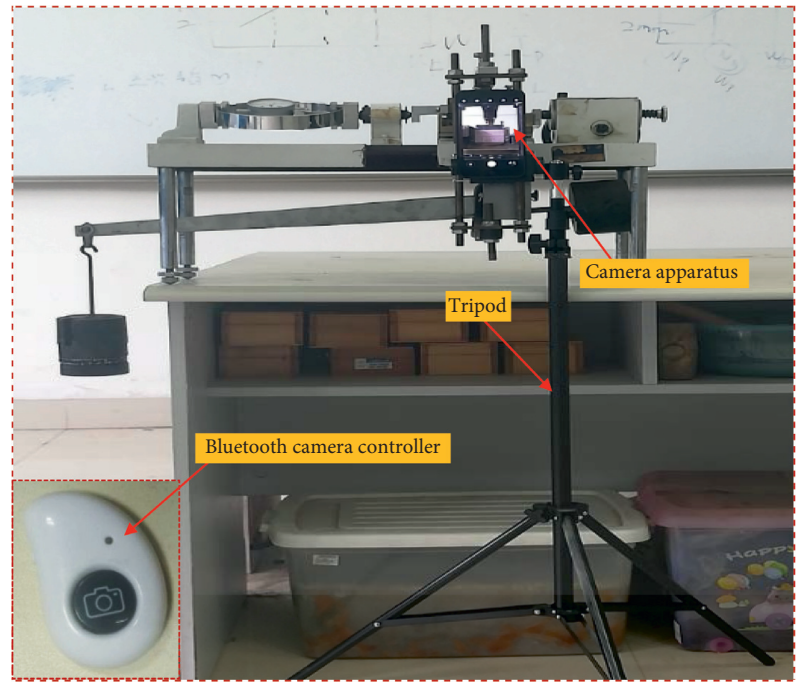

(b)

Figure 2: Test equipment. (a) A half-mold direct shear apparatus; (b) the image acquisition system.

raindrop method. The sample was carefully compacted to a height of $4.0 \mathrm{~cm}$ to fulfill the requirement of the degree of compaction.

(2) A weight with a certain mass $(5.1 \mathrm{~kg})$ was selected according to the lever ratio and the cross-sectional area of the sample. The sample was consolidated under the normal stress of $400 \mathrm{kPa}$ for 24 hours before shearing.

(3) The sample was sheared at the shear rate of $0.8 \mathrm{~mm} /$ min which is controlled by turning the handwheel at a constant speed of $1 / 15 \mathrm{r} / \mathrm{s}$, and the test was stopped at the displacement of $10.0 \mathrm{~mm}$.

The previous research results $[51,52]$ have shown that the accurate proportion between the pixel in the digital image and the physical length is essential for accurate measurement. The calibration test was carried out before the test with a ruler, which was placed on the surface of the plexiglass plate. It should be noted that once the shearing test was started, the camera should be fixed in position without any movement. Otherwise, the proportion between the pixel in the digital image and the physical length should be recalibrated.

\section{Results and Analysis}

3.1. Stress-Displacement Relationship. The shear stress-displacement curve of the sample under the normal stress of $400 \mathrm{kPa}$ is shown in Figure 3. It is found that the shear

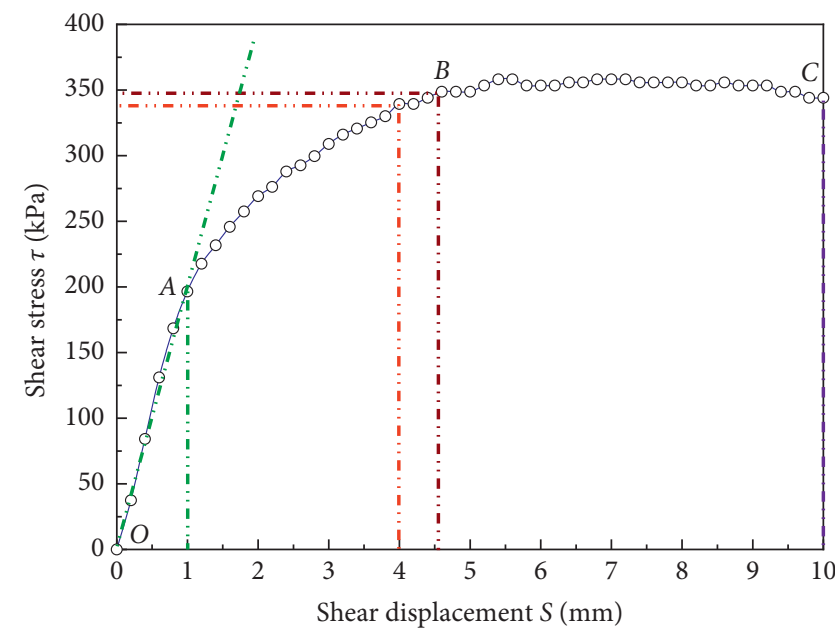

FIGURE 3: Shear stress-displacement curve from the half-mold direct shear test.

stress-displacement curve of the calcareous medium sand exhibits an overall trend of strain hardening. The shear stress-displacement curve could be divided into three stages: the linear elastic growth stage (OA region), the elastoplastic growth stage ( $\mathrm{AB}$ region), and the shear fracture or failure stage (BC region). The linear elastic growth stage (OA region) corresponds to the initial stage of the shear displacement within the range of $0-1.0 \mathrm{~mm}$. In this stage, the shear stress increases almost linearly with the shear 
TABle 2: Technical parameters of the image acquisition system.

\begin{tabular}{|c|c|c|c|c|c|c|}
\hline \multicolumn{2}{|c|}{ Camera } & \multicolumn{3}{|c|}{ Tripod } & \multicolumn{2}{|c|}{$\begin{array}{l}\text { Bluetooth camera } \\
\text { controller }\end{array}$} \\
\hline Resolution (pixel) & Image format & Material & Height adjustment range $(\mathrm{cm})$ & $\begin{array}{l}\text { Load capacity } \\
(\mathrm{kg})\end{array}$ & Control method & $\begin{array}{l}\text { Weight } \\
\text { (g) }\end{array}$ \\
\hline $12 \mathrm{MP}$ & JPG & Premium aluminium alloy & $43-136$ & 2 & Bluetooth & 100 \\
\hline
\end{tabular}

TABLE 3: Experimental design scheme.

\begin{tabular}{|c|c|c|c|c|c|c|c|c|c|}
\hline Sample & $\begin{array}{c}\text { Particle } \\
\text { size } D \\
(\mathrm{~mm})\end{array}$ & $\begin{array}{c}\text { Water } \\
\text { content } \omega \\
(\%)\end{array}$ & $\begin{array}{c}\text { Compactness } \\
(\%)\end{array}$ & $\begin{array}{c}\text { Cross- } \\
\text { sectional } \\
\text { area }\left(\mathrm{cm}^{2}\right)\end{array}$ & $\begin{array}{l}\text { Height } \\
(\mathrm{cm})\end{array}$ & $\begin{array}{l}\text { Normal } \\
\text { stress } \sigma_{n} \\
(\mathrm{kPa})\end{array}$ & Shear form & $\begin{array}{l}\text { Shear rate } \\
(\mathrm{mm} / \mathrm{min})\end{array}$ & $\begin{array}{c}\text { Termination } \\
\text { condition }\end{array}$ \\
\hline $\begin{array}{l}\text { Calcareous } \\
\text { sand }\end{array}$ & $0.5-1.0$ & 0 & 90 & 15 & 4 & 400 & $\begin{array}{l}\text { Consolidated- } \\
\text { quickly shearing }\end{array}$ & 0.8 & $S=10.0 \mathrm{~mm}$ \\
\hline
\end{tabular}

displacement and the shear modulus reaches $196.56 \mathrm{kPa}$. The elastoplastic growth stage ( $\mathrm{AB}$ region) which is within the shear displacement of $1.0-4.5 \mathrm{~mm}$ represents the intermediate stage of the whole shearing process. In this stage, the shear stress shows a plastic growth trend with the increasing shear displacement, and the increment of the shear stress is more and more slowly with the increasing shear displacement. For the BC region (shear displacement of $4.5-10.0 \mathrm{~mm}$ ), the shear stress fluctuates slightly with shear displacement. The deviation between the maximum and minimum shear stresses is only $3.92 \%$ of the maximum stress in this stage. The contact area of the calcareous sand between the upper and lower shear boxes has been completely fused, and the shearing process begins to fail. According to the Chinese National Standard for Soil Test Method (GB/T50123-2019) [53], the peak value of the shear stress-displacement curve should be adopted as the shear strength of the sample if the overall response of the sample is strain softening in the direct shear test. However, if the shear stress-displacement relationship shows strain hardening, the shear stress at the shear displacement of $4.0 \mathrm{~mm}$ should be taken as the shear strength of the sample. It could be found that the shear displacement $S=4.0 \mathrm{~mm}$ is still within the elastoplastic stage of the tested sample. It means that the shear strength which is represented by the shear stress at the shear displacement of $4.0 \mathrm{~mm}$ is inevitably smaller than the true shear strength of the sample.

3.2. Analysis of the Deformation Field. In this study, the digital photogrammetry software based on digital photographic deformation measurement (DPDM) technology developed by Li et al. $[26,27]$ was used to extract the mesostructural parameters of sand particles during shearing. The software system consists of the digital image analysis, named PhotoInfor, and the visual postprocessing named PostViewer. It provides powerful support for the observations of the whole process, the evolution, and the local analysis of the deformation of the sandstone, clay, reinforced concrete, rock, and other geotechnical materials based on the digital photographs [54]. It has been found that this digital photogrammetry software could also be applied for the measurement of the mesostructure parameters of granular materials [54-58]. Therefore, the digital photogrammetry software was employed to analyze the mesostructural parameters of the tested calcareous sand during the direct shear test. Before image processing, the image was numbered in sequence according to the shear displacement $(S)$ during the test. Then, the images were processed by the digital image analysis software by using the markerless deformation measurement mode. The results were processed by the postprocessing system PostViewer [26, 27]. In order to obtain accurate data of the mesostructure parameters of sand particles, it is necessary to convert the pixel values to the physical lengths by the BMP format images with the steel ruler scale as the reference to get the scale of the image, which is used for the extraction of the mesoscopic parameters of each image. The test parameters including analysis range, measurement interval, and search step size for the image analysis by PhotoInfor are summarized in Table 4 . The area for image analysis is shown in Figure 4.

The grid graphs for the deformation of calcareous sand during shearing were analyzed at three points which are selected from the $\mathrm{OA}, \mathrm{AB}$, and $\mathrm{BC}$ stages, respectively. The results are shown in Figure 5. It is observed that (1) the displacement of calcareous sand particles in the lower shear box is greater than that of the upper shear box. During shearing, the displacement is mainly concentrated on the sand particles in the lower shear box, while the magnitude and range of displacement of the sand particles in the upper shear box are almost unchanged. The upper shear box is fixed while the lower shear box moves by pushing the shaft, which allows the movement of the calcareous sand particles in the lower shear box towards the proving ringside at a uniform speed during shearing. Therefore, the amount of the displacement of the sand particles in the lower shear box is determined by the travel distance of the shear box. The sand particles in the upper shear box are moved by the dragging of the sand particles in the lower shear box so that their 
TABLE 4: Image analysis parameters used in PhotoInfor.

\begin{tabular}{|c|c|c|c|c|c|c|c|c|}
\hline Item & $\begin{array}{l}\text { Image pixels } \\
\text { (pixel×pixel) }\end{array}$ & $\begin{array}{l}\text { Image scale } \\
(\mathrm{mm} / \text { pixel })\end{array}$ & $\begin{array}{c}\text { Width of } \\
\text { analysis area } \\
\text { (pixel) }\end{array}$ & $\begin{array}{l}\text { Height of } \\
\text { analysis area } \\
\text { (pixel) }\end{array}$ & $\begin{array}{l}\text { Measurement point } \\
\text { interval (pixel) }\end{array}$ & $\begin{array}{l}\text { Number of } \\
\text { cells (unit) }\end{array}$ & $\begin{array}{l}\text { Half-length } \\
\text { of pixel block } \\
\text { (pixel) }\end{array}$ & $\begin{array}{l}\text { Search step } \\
\text { size (pixel) }\end{array}$ \\
\hline $\begin{array}{l}\text { Reference } \\
\text { value }\end{array}$ & $3936 \times 5248$ & 0.01763 & 1701 & 1134 & 45 & 988 & 30 & 0.01 \\
\hline
\end{tabular}

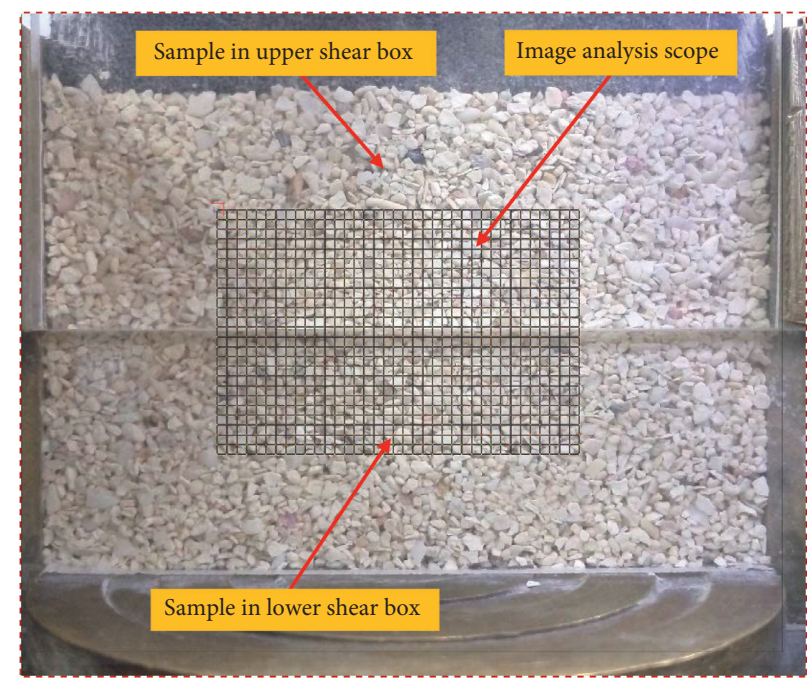

FIGURE 4: Image analysis area.

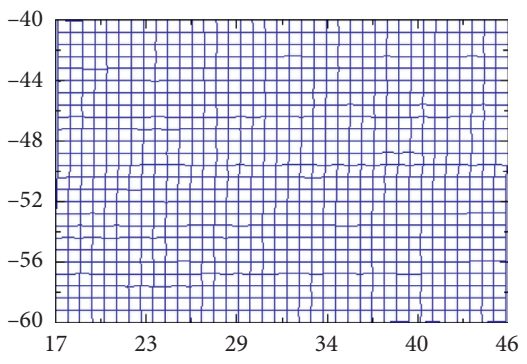

(a)

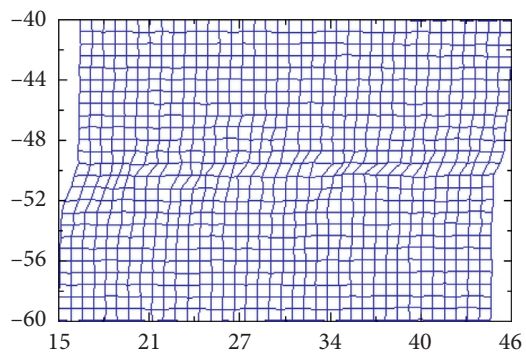

(b)

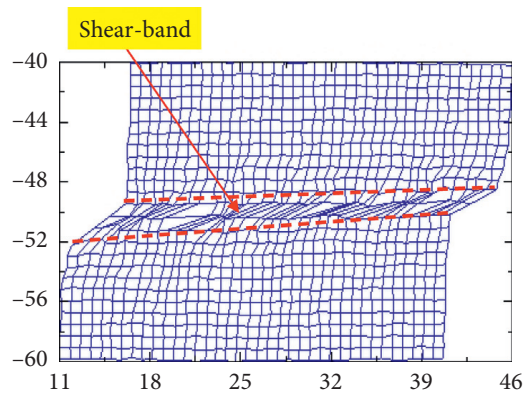

(c)

FIGURE 5: Grid graphs for the deformation of calcareous sand during shearing: (a) $S=1.0 \mathrm{~mm}$; (b) $S=3.0 \mathrm{~mm}$; (c) $S=7.8 \mathrm{~mm}$ (note: the units of horizontal and vertical coordinates in the figure are pixel numbers).

displacement is essentially determined by the shear force between two boxes. (2) The calcareous sand in the upper and lower shear boxes can interact with each other at the contact site, and the scope of the interaction is called shear-band. As the shear displacement increases, the shear-band becomes more obvious. The distortions of the grids at the junction of the two boxes shown in Figure 5(c) are induced by the large displacement of the particles in the shear-band. From those twisted grid lines, the location and thickness of the shear-band could be estimated. It is found that the thickness of the shearband of the tested sample subjected to the normal stress of $400 \mathrm{kPa}$ varies from $0.79 \mathrm{~mm}$ to $1.59 \mathrm{~mm}$. It is worth noting that the thickness of shear-band of calcareous sand in this study is obviously smaller than the previous research results [59-62], e.g., 5-10 times of median particle size $\left(d_{50}\right)$. The smaller direct shear box size may be the essential cause of this difference. (3) The shear-band range shown by the gridline in Figure 5(c) is a diagonal band from the lower left to the upper right. The particles involved in the shearing and experienced large displacement deformation are concentrated in this area. These results are consistent with those of $\mathrm{Li}$ et al. [49]. The shear process of fixing the upper shear box and moving the lower shear box is the main reason for this phenomenon.

The maximum shear strain of the sample varies with the displacement at each shear stage, as shown in Figure 6. The figures reproduce the development and evolution process of the shearband and the deformation mode of the calcareous sand during shearing. (1) At the initial stage of linear elastic deformation (OA region), the relative dislocation between the upper and lower shear boxes plays a compaction role in the sample. The sand particles 


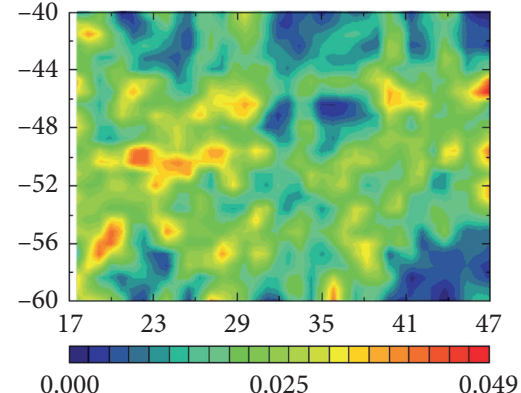

(a)

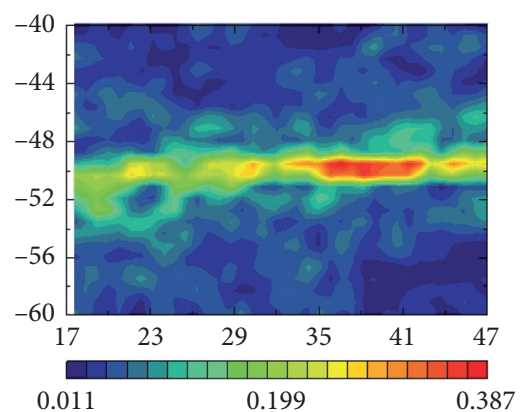

(d)

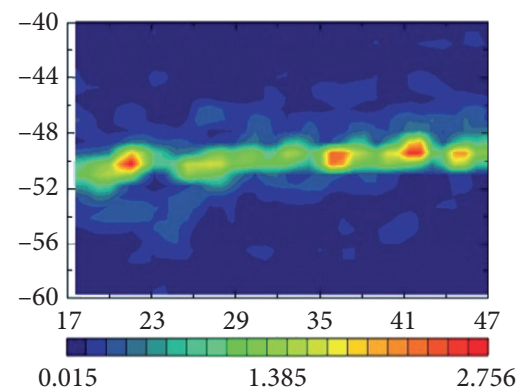

(g)

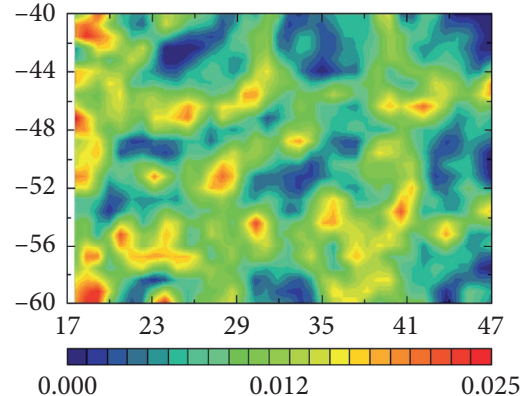

(b)

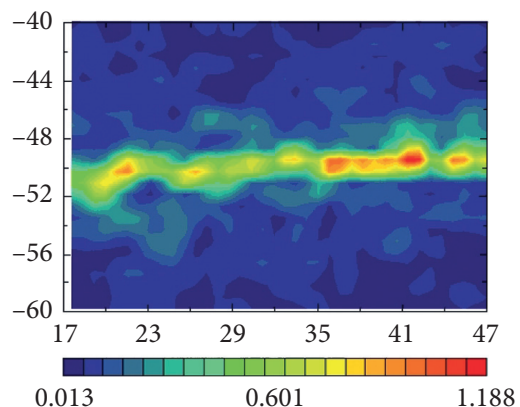

(e)

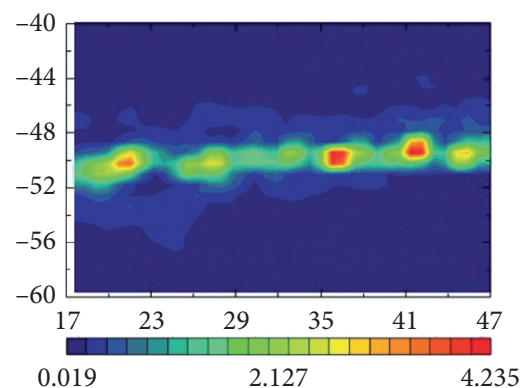

(h)

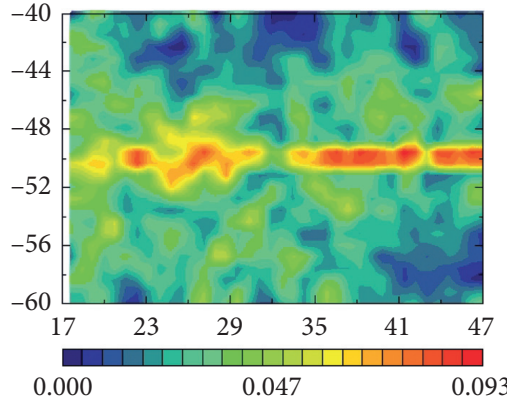

(c)

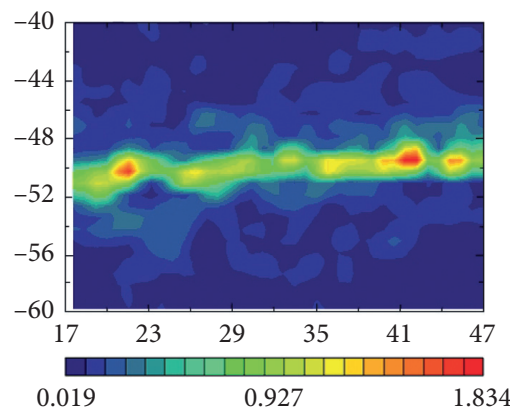

(f)

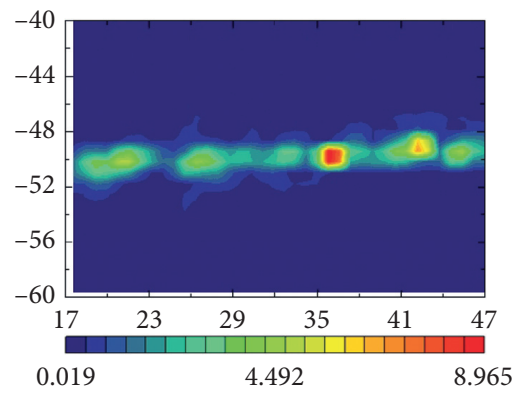

(i)

Figure 6: Distribution of maximum shear strain field in calcareous sand (note: the units of horizontal and vertical coordinates in the figure are pixel numbers). (a) $S=0.2 \mathrm{~mm}$, (b) $S=0.6 \mathrm{~mm}$, (c) $S=1.0 \mathrm{~mm}$, (d) $S=2.0 \mathrm{~mm}$, (e) $S=4.0 \mathrm{~mm}$, (f) $S=5.0 \mathrm{~mm}$, (g) $S=6.0 \mathrm{~mm}$, (h) $S=7.0 \mathrm{~mm}$, and (i) $S=8.4 \mathrm{~mm}$.

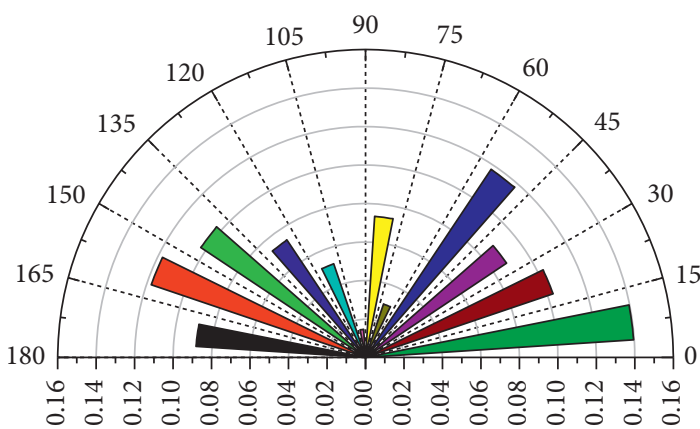

(a)

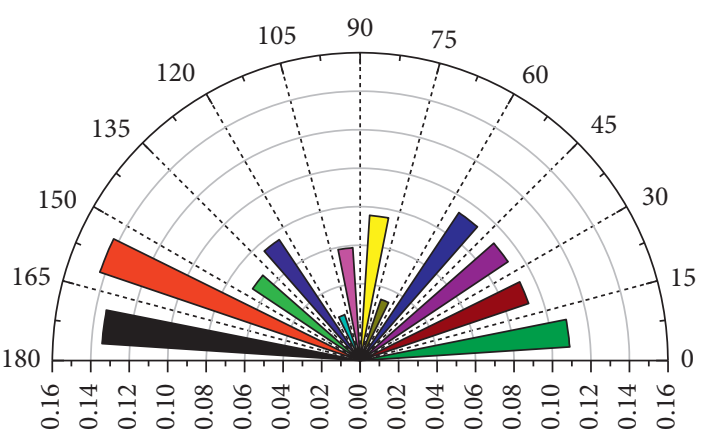

(b)

FIgURE 7: Rose plot for particle orientation distribution. (a) Preexperiment; (b) postexperiment.

adjust their positions constantly to adapt to the external changes, which result in larger local shear strains at a number of places in the sample. As the sample is adjusted to a more compact state, the relative motion between sand particles decreases, which results in the position where the larger local shear strain occurs also decreases. With the development of shear displacement; subsequently, the maximum shear strain concentrates at the junction of the two shear boxes and propagates from the two sides to the middle, and the 


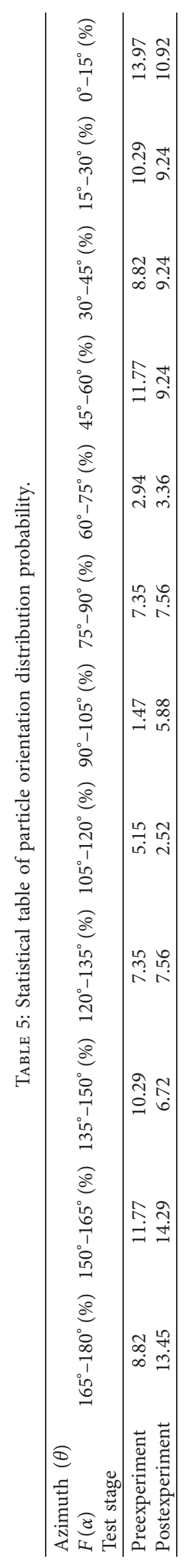


shear-band also becomes more prominent. (2) In the elastoplastic deformation stage ( $\mathrm{AB}$ region), the shear strain outside the shearband is further reduced with the increasing shear displacement, and the maximum shear strain is still within the shear-band. The relative motion between sand particles in the shear-band is intensified, and the development of the shear-band was more obvious. (3) In the plastic deformation stage (BC region), the shear strain outside the shear-band gradually disappears, and the maximum shear strain in the shear-band increases with the increasing shear displacement. The thickness of the shear-band is almost constant in this stage.

3.3. Azimuth Analysis. During shearing, the particles undergo both translational and rotational movement. The degree and trend of particle rotation are described by the change in the orientation arrangement of particles. The azimuth $\theta$, which is used for the statistical analysis of the particle orientation, is defined as the angle between the $X$-axis (horizontal) direction and the long axis of a particle. By observing the change in the movement patterns of the sand particles in the sample during shearing, it is found that the sand particles in the lower shear box exhibited more translational transformation than those in the upper shear box. The lower shear box moves forward with the rotation of the handwheel, while the position of the upper shear box does not change significantly due to the resistance by the proving ring. Therefore, the sand particles in the upper shear box could only counterbalance the external force during shearing via positional changes, and the probability of the particle rotation occurs is greater. The movement of the sand particles close to the shear-band in the upper shear box was analyzed. 100-150 sand particles from the images of the sample before and after shearing were randomly selected for profiling. The azimuth of the sand particles is measured quantitatively by the image analysis software (Image Pro Plus). In order to eliminate the effect of the number of sand particles on the test result, the probability of particle orientation distribution $F_{(\alpha)}$ was used. It is defined as follows:

$$
F(\alpha)=\frac{n_{i}}{n} \times 100 \%,
$$

where $n_{i}$ is the number of sand particles whose azimuth $\theta$ in the sample falls within a certain angle range, and $n$ is the total number of the sand particles. The test results are shown in Figure 7 and Table 5.

Figure 7 and Table 5 show the significant difference in the orientation distributions of the sand particles before and after the test. Before the test, the proportion of sand particles with azimuth near $90^{\circ}$ is significantly smaller; that is, the azimuths of the sand particles are roughly symmetrically distributed on both sides of $90^{\circ}$. The sand particles with a long axis aligning closely to the horizontal direction are more likely to be stable under normal stress, which forces the vertical particles tilt to both sides of $90^{\circ}$. As a result, the number of sand particles with an azimuth proximate to $90^{\circ}$ decreases, while the number of sand particles with an azimuth distributed on both sides of the $90^{\circ}$ increases.

During the direct shear test, the sand particles rotate under loading to consume the excess energy to maintain the equilibrium. It is found that the alignments of the sand particles changes after the test. The azimuths of the sand particles are mainly obtuse angles $\left(150-180^{\circ}\right)$, and the direction of the long axis of the sand particles is opposite to the direction of the shearband. The irregular particle shape of calcareous sand results in the interlocking of particles during shearing, and this interlocking works as the apparent cohesion, which is greater at the corners and smaller at the contact areas between smooth surfaces of the particles. The apparent cohesion reduces the energy of shear failure to a certain extent. In summary, during the direct shear test, the sand particles undergo four stages: random arrangement during sample preparation, compaction under normal stress, particle rotation during shearing, and ordered alignment after shearing.

\section{Conclusions}

In this study, the mesostructures of the calcareous sand particles at different stages of the direct shear test were investigated by the digital image processing technology. The following were found:

(1) Using digital photography together with image correlation analysis technology, the shearing deformation of the sample was quantitatively quantified. It meant that digital image processing technology was an effective method to observe the deformation modes and the evolution of geotechnical materials under shearing.

(2) The shear-band of the dry calcareous sand with a $90 \%$ degree of compaction under the normal stress of $400 \mathrm{kPa}$ was found to be approximately $0.79-1.59 \mathrm{~mm}$ in thickness and located at the junction of the upper and lower shear boxes.

(3) During the elastic deformation stage, the distribution of the shear strain was extremely uneven that the larger local shear strain occurred in some places of the sample. In the elastoplastic deformation stage, the position of the maximum shear strain gradually moved to the junction between the two shear boxes. In the plastic deformation stage, the maximum shear strain was concentrated in the centre of the shear-band. The range with larger local shear strain in the initial shear stage did not completely coincide with the shear-band.

(4) The azimuths of sand particles before shearing were roughly symmetrically distributed to both sides of $90^{\circ}$. However, after shearing, the azimuths of sand particles were mainly obtuse angles $\left(150-180^{\circ}\right)$, and the direction of the long axis of the sand particles tended to be opposite to the direction of the shear-band.

(5) During the direct shear test, the sand particles underwent four stages: random arrangement during sample preparation, compaction under normal stress, particle rotation during shearing, and ordered alignment after shearing.

\section{Data Availability}

Some or all data, models, or codes generated or used during the study are available from the corresponding author upon request. 


\section{Conflicts of Interest}

All the authors declare no conflicts of interest.

\section{Authors' Contributions}

All the authors have read and approved this version of the article, and due care has been taken to ensure the integrity of the work.

\section{Acknowledgments}

This work was funded by the National Natural Science Foundation of China (Nos. 41877271, 41372318, and 41772336) and the Strategic Priority Research Program of the Chinese Academy of Sciences (XDA13010301).

\section{References}

[1] X. Wang, J. Cui, Y. Wu, C. Q. Zhu, and X. Z. Wang, "Mechanical properties of calcareous silts in ahydraulic fill islandreef," Marine Georesources \& Geotechnology, 2020.

[2] Y. Wu, Y. Wang, and Q. Yang, "Experiment on drag force coefficient of calcareous sand in liquid considering the effect of particle shape," Rock and Soil Mechanics, vol. 39, no. 9, pp. 3203-3212, 2018.

[3] Y. Q. Wang, Y. Hong, Z. Guo, and L. Z. Wang, "Micro-and macro-mechanical behavior of crushable calcareous sand in South China Sea," Rock and Soil Mechanics, vol. 39, no. 1, pp. 199-215, 2018.

[4] Y. Shen, X. Shen, Y. M. Yu, H. L. Liu, H. Y. Ge, and X. X. Rui, "Macro-micro study of compressive deformation properties of calcareous sand with different particle fraction contents," Rock and Soil Mechanics, vol. 40, no. 10, pp. 3733-3740, 2019.

[5] Y. Wang, L. X. Zhou, and Q. Yang, "New drag coefficient model for irregular calcareous sand particles and its application into fluid-particle coupling simulation," Rock and Soil Mechanics, vol. 40, no. 5, pp. 2009-2015, 2019.

[6] X. Wang, Y. Wu, J. Cui, C.-Q. Zhu, and X.-Z. Wang, "Shape characteristics of coral sand from the South China Sea," Journal of Marine Science and Engineering, vol. 8, no. 10, p. 803, 2020.

[7] D. S. Xu, M. Huang, and Y. Zhou, "One-dimensional compression behavior of calcareous sand and marine clay mixtures," International Journal of Geomechanics, ASCE, vol. 20, Article ID 04020137, 2020.

[8] D.-S. Xu, H.-B. Liu, R. Rui, and Y. Gao, "Cyclic and postcyclic simple shear behavior of binary sand-gravel mixtures with various gravel contents," Soil Dynamics and Earthquake Engineering, vol. 123, pp. 230-241, 2019.

[9] D. Xu, Z. Tang, and L. Zhang, "Interpretation of coarse effect in simple shear behavior of binary sand-gravel mixture by DEM with authentic particle shape," Construction and Building Materials, vol. 195, pp. 292-304, 2019.

[10] M. Oda and J. Konishi, "Microscopic deformation mechanism of granular material in simple shear," Soils and Foundations, vol. 14, no. 4, pp. 25-38, 1974.

[11] D.-S. Xu, X.-Y. Xu, W. Li, and B. Fatahi, "Field experiments on laterally loaded piles for an offshore wind farm," Marine Structures, vol. 69, Article ID 102684, 2020.

[12] J. D. Frost and C. Y. Kuo, "Automated determination of the distribution of local void ratio from digital images," Geotechnical Testing Journal, vol. 19, no. 2, pp. 107-117, 1996.
[13] C. Y. Kuo, J. D. Frost, and J. L. A. Chameau, "Image analysis determination of stereology based fabric tensors," Géotechnique, vol. 48, no. 4, pp. 515-525, 1998.

[14] S. Nemat-Nasser and N. Okada, "Radiographic and microscopic observation of shear bands in granular materials," Géotechnique, vol. 51, no. 9, pp. 753-765, 2001.

[15] J. Otani, T. Mukunoki, and Y. Obara, "Application of X-ray CT method for characterization of failure in soils," Soils and Foundations, vol. 40, no. 2, pp. 111-118, 2000.

[16] J. B. Ye, J. F. Zhang, and W. L. Zou, "Influences of grain shape on pore characteristics of filled breakstone aggregate," Rock and Soil Mechanics, vol. 39, no. 12, pp. 4457-4467, 2018.

[17] M. M. Darrow and R. M. Lieblappen, "Visualizing cation treatment effects on frozen clay soils through $\mu \mathrm{CT}$ scanning," Cold Regions Science and Technology, vol. 175, Article ID 103085, 2020.

[18] L. Kong, F. X. Chen, and J. Li, "Meso-direct-shear test of sand based on digital image correlation method and its PFC numerical simulation," Rock and Soil Mechanics, vol. 34, no. 10, pp. 2971-2978, 2013.

[19] S. Liu, F. T. S. Chan, and W. Ran, "Decision making for the selection of cloud vendor: an improved approach under group decision-making with integrated weights and objective/subjective attributes," Expert Systems with Applications, vol. 55, pp. 37-47, 2016.

[20] M. Xu, T. Li, Z. Wang, X. Deng, R. Yang, and Z. Guan, "Reducing complexity of HEVC: a deep learning approach," IEEE Transactions on Image Processing, vol. 27, no. 10, pp. 5044-5059, 2018.

[21] T. Li, M. Xu, C. Zhu, R. Yang, Z. Wang, and Z. Guan, “A deep learning approach for multi-frame in-loop filter of HEVC," IEEE Transactions on Image Processing, vol. 28, no. 11, pp. 5663-5678, 2019.

[22] C. Li, M. Xu, S. Y. Zhang, and P. L. Callet, "State-of-the-art in 360 degrees video/image processing: perception, assessment and compression," IEEE Journal of Selected Topics in Signal Processing, vol. 14, no. 1, pp. 5-26, 2020.

[23] Q. Zhu, "Research on road traffic situation awareness system based on image big data," IEEE Intelligent Systems, vol. 35, no. 1, pp. 18-26, 2020.

[24] K. A. Alshibli and S. Sture, "Sand shear band thickness measurements by digital imaging techniques," Journal of Computing in Civil Engineering, vol. 13, no. 2, pp. 103-109, 1999.

[25] D. J. White, W. A. Take, and M. D. Bolton, "Measuring soil deformation in geotechnical models using digital images and PIV analysis," in Proceedings of the 10th International Conference on Computer Methods and Advances in Geotechanics, pp. 997-1002, Tueson, AZ, USA, January 2001.

[26] Y. H. Li, H. H. Zhu, U. Katsutoshi, and M. Akitoshi, "Deformation field measurement for granular soil model using image analysis," Chinese Journal of Geotechnical Engineering, vol. 26, no. 1, pp. 36-41, 2004.

[27] Y. H. Li, H. W. Jing, and Q. Y. Zeng, "Development and application of digital photogrammetry software package for geotechnical engineering," Chinese Journal of Rock Mechanics and Engineering, vol. 25, no. S2, pp. 3859-3866, 2006.

[28] J. Zhou, R. C. Yu, and M. C. Jia, "Measurement of microstructure parameters for granular soil model using digital image technology," Chinese Journal of Geotechnical Engineering, vol. 28, no. 12, pp. 2047-2052, 2006.

[29] J. Zhou, D. D. Shi, F. Wu, and J. F. Xue, "Visualized cyclic triaxial tests on sand liquefaction using digital imaging 
technique," Chinese Journal of Geotechnical Engineering, vol. 33, no. 1, pp. 81-87, 2011.

[30] G. Zhang, J. M. Zhang, and D. F. Liang, "Measurement of soil particle movement in soil-structure interface test," Chinese Journal of Geotechnical Engineering, vol. 27, no. 8, pp. 903907, 2005.

[31] L. T. Shao, Y. Z. Sun, Z. P. Wang, and Y. L. Liu, “Application of digital image processing technique to triaxial test in soil mechanics," Rock and Soil Mechanics, vol. 27, no. 1, pp. 29-34, 2006.

[32] W. B. Liu and Q. Tian, "Analysis of soil meso-structure under uplift load," Chinese Journal of Rock Mechanics and Engineering, vol. 26, no. S2, pp. 4311-4318, 2007.

[33] X. Cheng, L. He, H. Lu, Y. Chen, and L. Ren, "Optimal water resources management and system benefit for the Marcellus shale-gas reservoir in Pennsylvania and West Virginia," Journal of Hydrology, vol. 540, pp. 412-422, 2016.

[34] D.-B. Zhang, Y. Zhang, T. Cheng et al., "Measurement of displacement for open pit to underground mining transition using digital photogrammetry," Measurement, vol. 109, pp. 187-199, 2017.

[35] P. V. Divya, B. V. S. Viswanadham, and J. P. Gourc, "Centrifuge model study on the performance of fiber reinforced clay-based landfill covers subjected to flexural distress," Applied Clay Science, vol. 142, no. S1, pp. 173-184, 2017.

[36] R. Pang, B. Xu, X. Kong, and D. Zou, "Seismic fragility for high CFRDs based on deformation and damage index through incremental dynamic analysis," Soil Dynamics and Earthquake Engineering, vol. 104, pp. 432-436, 2018.

[37] S. A. Safavizadeh, A. Wargo, and Y. R. Kim, "Utilizing digital image correlation (DIC) in asphalt pavement testing," Journal of Testing and Evaluation, vol. 46, no. 3, pp. 984-998, 2018.

[38] Q. Lyu, X. Long, P. G. Ranjith, J. Tan, and Y. Kang, "Experimental investigation on the mechanical behaviours of a low-clay shale under water-based fluids," Engineering Geology, vol. 233, pp. 124-138, 2018.

[39] A. J. Bowman and S. K. Haigh, "Subsurface deformation mechanisms beneath a flexible pavement using image correlation," Géotechnique, vol. 69, no. 7, pp. 627-637, 2019.

[40] J. Wang, W. Liu, W. W. Chen, X. B. Ran, Y. F. Zhang, and G. C. Lin, "An improved method for quantifying liquid saturation using transparent soil," Advances in Civil Engineering, vol. 2019, Article ID 1867342, 2019.

[41] B. Mou, X. Li, Q. Qiao, B. He, and M. Wu, "Seismic behaviour of the corner joints of a frame under biaxial cyclic loading," Engineering Structures, vol. 196, Article ID 109316, 2019.

[42] C. J. Liu, X. W. Deng, J. Liu, T. J. Peng, S. P. Yang, and Z. L. Zheng, "Dynamic response of saddle membrane structure under hail impact," Engineering Structures, vol. 214, Article ID 110597, 2020.

[43] H. N. Yu, S. H. Shen, G. P. Qian, and X. B. Gong, "Packing theory and volumetrics-based aggregate gradation design method," Journal of Materials in Civil Engineering, vol. 32, no. 6, Article ID 04020110, 2020.

[44] B. Xu, R. Pang, and Y. Zhou, "Verification of stochastic seismic analysis method and seismic performance evaluation based on multi-indices for high CFRDs," Engineering Geology, vol. 264, Article ID 105412, 2020.

[45] Z. Li, H. Zhou, D. W. Hu, and C. Q. Zhang, "Yield criterion for rocklike geomaterials based on strain energy and CMP model," International Journal of Geomechanics, vol. 20, no. 3, Article ID 04020013, 2020.

[46] Y. Chen, J. Li, H. Lu, and P. Yan, "Coupling system dynamics analysis and risk aversion programming for optimizing the mixed noise-driven shale gas-water supply chains," Journal of Cleaner Production, vol. 278, Article ID 123209, 2021.

[47] W. B. Liu, H. Zhang, L. Cao, J. Zhou, and D. D. Shi, "Mesostructure direct shear apparatus," Chinese Patent NO. 200820156414.3, 2008.

[48] X. Wang, X.-Z. Wang, C.-Q. Zhu, and Q.-S. Meng, "Shear tests of interfaces between calcareous sand and steel," Marine Georesources \& Geotechnology, vol. 37, no. 9, pp. 1095-1104, 2019.

[49] X. Wang, C.-Q. Zhu, and X.-Z. Wang, "Experimental study on the coefficient of lateral pressure at rest for calcareous soils," Marine Georesources \& Geotechnology, vol. 38, no. 8, pp. 989-1001, 2020.

[50] X. Wang, C.-Q. Zhu, X.-Z. Wang, and Y. Qin, "Study of dilatancy behaviors of calcareous soils in a triaxial test," Marine Georesources \& Geotechnology, vol. 37, no. 9, pp. 1057-1070, 2019.

[51] X. P. Zhou, Y. J. Lian, L. N. Y. Wong, and F. Berto, "Understanding the fracture behavior of brittle and ductile multiflawed rocks by uniaxial loading by digital image correlation," Engineering Fracture Mechanics, vol. 199, pp. 438-460, 2018.

[52] X. P. Zhou, Y. T. Wang, J. Z. Zhang, and F. N. Liu, "Fracturing behavior study of three-flawed specimens by uniaxial compression and $3 \mathrm{D}$ digital image correlation: sensitivity to brittleness," Rock Mechanics and Rock Engineering, vol. 52, no. 8, pp. 691-718, 2019.

[53] GB/T50123-2019, Standard for Soil Test Method, National Standards of the People's Republic of China, Beijing, China, 2019.

[54] Y. H. Li, H. W. Jing, H. H. Zhu, and X. F. Ma, "Experimental investigation on progressive deformation patterns of sand foundation in centrifuge test using digital photogrammetry," Chinese Journal of Geotechnical Engineering, vol. 28, no. 3, pp. 306-311, 2006.

[55] Y. H. Li, "Study on digital photography-based deformation measurement technique and its application in geo-physical model experiment," Chinese Journal of Rock Mechanics and Engineering, vol. 24, no. 7, p. 1273, 2005.

[56] Y. H. Li, H. W. Jing, H. H. Zhu, and U. Katsutoshi, "A technique of identifying shear band accurately in granular soil using image correlation analysis," Rock and Soil Mechanics, vol. 28, no. 3, pp. 522-526, 2007.

[57] Y. H. Li, H. H. Zhu, H. W. Jing, and U. Katsutoshi, "Experimental investigation of shear deformation patterns in sands based on digital image correlation," Journal of Tongji University (Natural Science), vol. 35, no. 5, pp. 685-689, 2007.

[58] L. Cao, W. B. Liu, X. Z. Li, X. B. Zhao, and Y. X. Li, "Experimental study of compression deformation patterns in sands based on digital image analysis," Rock and Soil Mechanics, vol. 33, no. 4, pp. 1018-1024, 2012.

[59] I. Vardoulakis, M. Goldscheider, and G. Gudehus, "Formation of shear bands in sand bodies as a bifurcation problem," International Journal for Numerical and Analytical Methods in Geomechanics, vol. 2, no. 2, pp. 99-128, 1978.

[60] R. J. Finno, W. W. Harris, and G. Viggiani, "Shear bands in plane strain compression of loose sand," Géotechnique, vol. 47, no. 1, pp. 149-165, 1997.

[61] S. Nemat-Nasser and N. Okada, "Strain localization in particulate media," in Proceedings of the 12th Engineering Mechanics Conference, LA Jolla, CA, USA, May 1998.

[62] T. Francesco, W. Jack, and A. Edward, "Observing strain localisation processes in BIOR cemented sand using x-ray imaging," Granular Matter, vol. 13, no. 3, pp. 247-250, 2011. 